\title{
Chemical trends in the Galactic halo from APOGEE data
}

\author{
E. Fernández-Alvar, ${ }^{1 \star}$ L. Carigi,${ }^{1}$ C. Allende Prieto, ${ }^{2,3}$ M. R. Hayden, ${ }^{4}$ T. C. Beers,${ }^{5}$ \\ J. G. Fernández-Trincado, ${ }^{6}$ A. Meza,${ }^{7}$ M. Schultheis, ${ }^{4}$ B. X. Santiago, ${ }^{8,9}$ \\ A. B. Queiroz, ${ }^{8,9}$ F. Anders, ${ }^{9,10}$ L. N. da Costa ${ }^{9,11}$ and C. Chiappini ${ }^{9,10}$ \\ ${ }^{1}$ Instituto de Astronomía, Universidad Nacional Autónoma de México, Apartado Postal 70-264, Ciudad Universitaria, Ciudad de México 04510, México \\ ${ }^{2}$ Instituto de Astrofísica de Canarias, Vía Láctea, E-38205 La Laguna, Tenerife, Spain \\ ${ }^{3}$ Departamento de Astrofísica, Universidad de La Laguna, E-38206 La Laguna, Tenerife, Spain \\ ${ }^{4}$ Laboratoire Lagrange (UMR7293), Universite de Nice Sophia Antipolis, CNRS, Observatoire de la Cote dAzur, BP 4229, F-06304 Nice Cedex 4, France \\ ${ }^{5}$ Department of Physics and JINA Center for the Evolution of the Elements, University of Notre Dame, Notre Dame, IN 46556, USA \\ ${ }^{6}$ Institut Utinam, CNRS UMR 6213, Université de Franche-Comté, OSU THETA Franche-Comté-Bourgogne, Observatoire de Besançon, BP 1615, F-25010 \\ Besançon Cedex, France \\ ${ }^{7}$ Departamento de Ciencias Fisicas, Universidad Andres Bello, Sazie 2212, Santiago, Chile \\ ${ }^{8}$ Instituto de Física, Universidade Federal do Rio Grande do Sul, Caixa Postal 15051, 91501-970 Porto Alegre, Brazil \\ ${ }^{9}$ Laboratório Interinstitucional de e-Astronomia-LIneA, Rua Gal. José Cristino 77, 20921-400 Rio de Janeiro, Brazil \\ ${ }^{10}$ Leibniz-Institut fur Astrophysik Potsdam (AIP), An der Sternwarte 16, D-14482 Potsdam, Germany \\ ${ }^{11}$ Observatório Nacional, Rua Gal. José Cristino 77, 20921-400 Rio de Janeiro, Brazil
}

Accepted 2016 November 2. Received 2016 November 1; in original form 2016 July 25

\begin{abstract}
A BSTRA C T
The galaxy formation process in the $\Lambda$ cold dark matter scenario can be constrained from the analysis of stars in the Milky Way's halo system. We examine the variation of chemical abundances in distant halo stars observed by the Apache Point Observatory Galactic Evolution Experiment (APOGEE), as a function of distance from the Galactic Centre $(r)$ and iron abundance $([M / \mathrm{H}])$, in the range $5 \lesssim r \lesssim 30 \mathrm{kpc}$ and $-2.5<[M / \mathrm{H}]<0.0$. We perform a statistical analysis of the abundance ratios derived by the APOGEE pipeline (ASPCAP) and distances calculated by several approaches. Our analysis reveals signatures of a different chemical enrichment between the inner and outer regions of the halo, with a transition at about $15 \mathrm{kpc}$. The derived metallicity distribution function exhibits two peaks, at $[\mathrm{M} / \mathrm{H}] \sim-1.5$ and $\sim-2.1$, consistent with previously reported halo metallicity distributions. We obtain a difference of $\sim 0.1$ dex for $\alpha$-element-to-iron ratios for stars at $r>15 \mathrm{kpc}$ and $[\mathrm{M} / \mathrm{H}]>-1.1$ (larger in the case of $\mathrm{O}, \mathrm{Mg}$, and $\mathrm{S}$ ) with respect to the nearest halo stars. This result confirms previous claims for low- $\alpha$ stars found at larger distances. Chemical differences in elements with other nucleosynthetic origins $(\mathrm{Ni}, \mathrm{K}, \mathrm{Na}$, and $\mathrm{Al}$ ) are also detected. $\mathrm{C}$ and $\mathrm{N}$ do not provide reliable information about the interstellar medium from which stars formed because our sample comprises red giant branch and asymptotic giant branch stars and can experience mixing of material to their surfaces.
\end{abstract}

Key words: stars: abundances - Galaxy: halo-Galaxy: stellar content.

\section{INTRODUCTION}

The $\Lambda$ cold dark matter paradigm predicts that galaxies form hierarchically from mergers of lower mass subsystems. Numerical simulations of the formation of Milky Way-like galaxies based on this scenario (e.g. Tissera et al. 2014, and references therein) predict that the halo of our Milky Way is expected to comprise at least two diffuse stellar components with differing spatial distribu-

^E-mail: emma@astro.unam.mx tions, chemistry, and kinematics, along with a number of individual overdensities and stellar debris streams. A large body of recent observations of the Milky Way and external galaxies provide evidence supporting this model. In particular, the Milky Way's stellar halo has been found to be far from homogeneous (Belokurov et al. 2009), with a metallicity distribution function (MDF) which differs between the inner- and outer-halo regions (Carollo et al. 2007, 2010; Beers et al. 2012; Allende Prieto et al. 2014). Chen et al. (2014) and Janesh et al. (2016) have found similar results based on in situ samples of distant giants in the halo. Analyses of relatively local samples of halo stars with photometric metallicity determinations 
(e.g. An et al. 2013, 2015), combined with available proper motions, have also indicated the presence of significant numbers of stars from the outer-halo population at distances within $\sim 10 \mathrm{kpc}$ of the Sun.

A dichotomy in the $\alpha$-element-to-iron ratios, $[\alpha / \mathrm{Fe}]$, for stars with halo kinematics has also been identified in the solar neighbourhood (Fulbright 2002; Gratton et al. 2003; Ishigaki, Chiba \& Aoki 2010; Nissen \& Schuster 2010, 2011). Since the $\alpha$-elements and $\mathrm{Fe}$ are primarily produced by different stellar progenitors, their relative abundances can provide constraints on the nature of the previous generations of stellar populations, such as the initial mass function (IMF), the star formation rate (SFR), and the efficiency of star formation in different environments, all of which affect the production and ejection of these elements to the interstellar medium (ISM).

In particular, the $\alpha$-elements are synthesized and expelled mainly by massive stars in the pre-supernova and supernova stages (Type II supernovae, $\mathrm{SNeII}$ ), and $\mathrm{Fe}$ is largely produced and driven out by binaries involving low- and intermediate-mass stars (LIMS) during their last stages of evolution (Type Ia supernova, SNeIa). Different chemical patterns point to stars born in environments with different IMFs and SFRs. Thus, chemical analysis of the halo stellar populations can provide information on the Galactic formation processes.

The advent of large surveys allows us to better characterize the properties of the stellar populations in the Galaxy. Previous studies were performed based on samples of a few hundred halo stars in a local volume. By contrast, current surveys, such as the Sloan Digital Sky Survey (SDSS; York et al. 2000; Alam et al. 2015), provide data for hundreds of thousands of stars throughout the halo of the Milky Way. Specific programmes to investigate the Galaxy have been included in SDSS and its extensions. The most recent subsurvey of this type is the Apache Point Observatory Galactic Evolution Experiment (APOGEE; Majewski et al. 2015). This programme has observed $\sim 150000$ stars for which stellar parameters and chemical abundances have been determined. Analysis of these high-quality data has already confirmed the $[\alpha / \mathrm{Fe}]$ dichotomy, exploring nearby halo stars in the metallicity range $-1.2<[\mathrm{Fe} / \mathrm{H}]^{1}<-0.55$ (Hawkins et al. 2015).

The SDSS stellar surveys explore the Galaxy over a broad range of distances, up to $\sim 100 \mathrm{kpc}$ from the Galactic Centre. The aforementioned studies inferred halo properties from stars identified by their local kinematics; the new data permit investigation of the properties of the Galactic halo identified by location in the Galaxy. Analyses of in situ halo stars can provide more complete information about the halo as a function of distance.

Fernández-Alvar et al. (2015, hereafter FA15) determined elemental abundances from low-resolution optical stellar spectra in the SDSS data base, comprising (i) observations from the original SDSS project and data from the Sloan Extension for Galactic Understanding and Exploration (SEGUE) programme (Yanny et al. 2009) and its extension (SEGUE-2), and (ii) spectrophotometric calibrators from the Baryon Oscillation Spectroscopic Survey (Dawson et al. 2013). This paper examined the variation of $[\mathrm{Fe} / \mathrm{H}],[\mathrm{Ca} / \mathrm{H}]$, and $[\mathrm{Mg} / \mathrm{H}]$ as a function of distance from the Galactic Centre, $r$, as well as the $[\mathrm{Ca} / \mathrm{Fe}]$ and $[\mathrm{Mg} / \mathrm{Fe}]$ abundance ratios as a function of $r$ and $[\mathrm{Fe} / \mathrm{H}]$. Chemical gradients were detected for these three elements, as well as variations in the $[\mathrm{Ca} / \mathrm{Fe}]$ and $[\mathrm{Mg} / \mathrm{Fe}]$ versus $[\mathrm{Fe} / \mathrm{H}]$ behaviours as a function of $r$, pointing to different $\alpha$-element enrichment histories for the inner- and outer-halo regions. In this

${ }^{1}[X / \mathrm{H}]=\log _{10}\left(\frac{N(X)}{N(\mathrm{H})}\right)-\log _{10}\left(\frac{N(X)}{N(\mathrm{H})}\right) \odot$. paper, analysis of higher quality data from APOGEE enables an independent assessment of these trends based on improved stellar parameters and chemical abundances.

This paper is organized as follows. Section 2 provides a brief description of the APOGEE data. Section 3 describes how we selected our in situ halo sample, the stellar parameters, and abundances determined by the APOGEE Stellar Parameters and Chemical Abundances Pipeline (ASPCAP), the available distance estimates for APOGEE stars, and the methods used to determine the chemical trends across the halo system. Section 4 presents our results, which are described in more detail in Section 5. Finally, we summarize our main conclusions in Section 6.

\section{OBSERVATIONS}

Our analysis was performed making use of the DR12 data products for APOGEE observations taken between 2011 September and 2014 July (Eisenstein et al. 2011; Majewski et al. 2015; Nidever et al. 2015). Using the same $2.5 \mathrm{~m}$ telescope at Apache Point Observatory as that employed for previous SDSS projects (Gunn et al. 2006), APOGEE is a Galactic survey designed to obtain infrared stellar spectra in the $H$ band $(1.5-1.7 \mu \mathrm{m})$ with a resolving power of $R \sim 22500$. From such spectra, stellar atmospheric parameters and chemical abundances of 15 elements $(C, N$, $\mathrm{O}, \mathrm{Na}, \mathrm{Mg}, \mathrm{Al}, \mathrm{Si}, \mathrm{S}, \mathrm{K}, \mathrm{Ca}, \mathrm{Ti}, \mathrm{V}, \mathrm{Mn}, \mathrm{Fe}, \mathrm{Ni}$ ) were determined with the ASPCAP pipeline (Holtzman et al. 2015; García Pérez et al. 2016). APOGEE was designed to explore the principal stellar components of the Galaxy, mainly the Galactic disc and bulge, but it also observed stars which are members of the Galactic halo. Halo stars were targeted following the same general colour-cut criteria, $(J-K)_{0}>0.5$ as all APOGEE observations. Halo targets in APOGEE lie mainly at Galactic latitudes $b>16^{\circ}$. For further details regarding the target selection in APOGEE, see Zasowski et al. (2013).

\section{ANALYSIS}

The aim of this work is to evaluate the variation of elemental abundances across the Galactic halo, using in situ halo stars out to the largest distances reached by the APOGEE observations, $\sim 20-30 \mathrm{kpc}$ from the Galactic Centre.

\subsection{Sample}

We first remove stars from our sample with unreliable stellar parameters and chemical abundance estimates, taking into account the flags provided in the data files which indicate suspicious ASPCAP results and/or instrumental issues (see Holtzman et al. 2015). Specifically, we reject those stars in the data base for which the STAR_BAD bit flag in the ASPCAPFLAG bitmask is set. This flag warns about stars with unreliable $T_{\text {eff }}$ and $\log g$ estimates, bad matches to synthetic spectra in the ASPCAP analysis, signal-tonoise ratios $(\mathrm{S} / \mathrm{N})$ per pixel in the final combined spectrum lower than 50, and/or the cases in which the spectrum exhibits broad lines likely due to significant stellar rotation. In addition, we do not consider the spectra of stars with the GRIDEDGE_BAD flag set in the ELEMFLAG bitmask, which correspond to those stars for which the resulting abundance estimate is closer than $1 / 8$ th of the grid spacing to the edge of grid (see García Pérez et al. 2016). Finally, we also avoid stars with spectra affected by persistence in the detectors (which may lead to significant errors in stellar parameters and abundance determination - see section 5.7 in Holtzman et al. 2015), by considering only stars which do not 
have the PERSIST_LOW, PERSIST_MED, and PERSIST_HIGH flags set in the STARFLAG bitmask. For more details about APOGEE flags, we refer the interested reader to the web page http://www.sdss.org/dr12/algorithms/bitmasks/.

Besides the selection criteria discussed above, we also apply other restrictions in $T_{\text {eff }}$ and $\log g$ which can arise from issues described by Holtzman et al. (2015). We only consider stars with estimated $T_{\text {eff }}>4000 \mathrm{~K}$, because at cooler temperatures the quality of the ASPCAP fitting is significantly lower. The calibration performed to the $\log g$ FERRE outputs, by comparing with asteroseismic $\log g$ estimates for stars observed by APOGEE in the Kepler field (Pinsonneault et al. 2014), shows that stars at $\log g \geq 4$ deviate considerably from asteroseismic gravities (Holtzman et al.). Therefore, they only calibrated data with lower $\log g$ estimates. Thus, we only consider stars with surface gravity estimates in the range $1.0<\log g<3.5$. In addition, we reject stars which were targeted as belonging to open or globular clusters, since we are interested in the chemical analysis of halo field stars; stars in clusters can exhibit chemical patterns which differ from those observed in field stars (see, e.g., Lind et al. 2015; Fernández-Trincado et al. 2016).

Finally, in addition to the existing target selection criteria for APOGEE observations, we select our halo sample by considering objects with derived distances from the Galactic plane $|z|>5 \mathrm{kpc}$. The resulting sample comprises a total of $\sim 400$ stars.

In order to check whether our sample comprises only stars belonging to the halo, we also inspect their kinematics. For this purpose, we derive the full space velocities with respect to the local standard of rest, $V_{\text {tot }}$, using the radial velocities provided by DR12 and proper motions from UCAC4 ${ }^{2}$ (Zacharias et al. 2013). Stars with $V_{\text {tot }}>$ $180 \mathrm{kms}^{-1}$ are usually considered to belong to the halo. Our sample includes some stars with lower $V_{\text {tot }}$. It is not clear why some of these stars have such low velocity values. One possibility is that, at a few kiloparsecs from the Sun, the UCAC4 proper motion uncertainties are similar to or greater than the intrinsic proper motions (see section 6.3 in Bovy et al. 2014). These uncertainties propagate to the derived velocities, introducing large errors. After having checked that excluding these stars does not significantly impact our results, we have decided to retain them in our sample.

The top panel in Fig. 1 shows the MDF for our final sample of stars, which is discussed in Section 4.1. We note that our MDF is in agreement with previous MDFs derived for halo samples (Carollo et al. 2007, 2010; An et al. 2013, 2015; Allende Prieto et al. 2014; Chen et al. 2014), displaying a maximum at $[M / \mathrm{H}] \sim-1.5$ and a secondary peak at lower $[M / \mathrm{H}](\sim-2.1)$. We conclude that our sample is comprised almost entirely of bona fide halo stars.

\subsection{Stellar parameters and chemical abundances}

The basic techniques followed in ASPCAP for stellar parameter and chemical abundance determination are the same as in the analysis performed by FA15 - comparison of the observed spectrum with a library of synthetic spectra covering a range of stellar parameters, looking for the parameter combination which returns the lowest $\chi^{2}$. This comparison is performed using the code FERRE $^{3}$ (Allende Prieto et al. 2006). The analysis proceeds in two steps:

(i) the stellar parameters $T_{\text {eff }}$ and $\log g$ are determined from a search fitting the entire available spectral range, and

\footnotetext{
${ }^{2}$ http://www.usno.navy.mil/USNO/astrometry/optical-IR-prod/ucac

${ }^{3}$ FERRE is available from http://hebe.as.utexas.edu/ferre
}
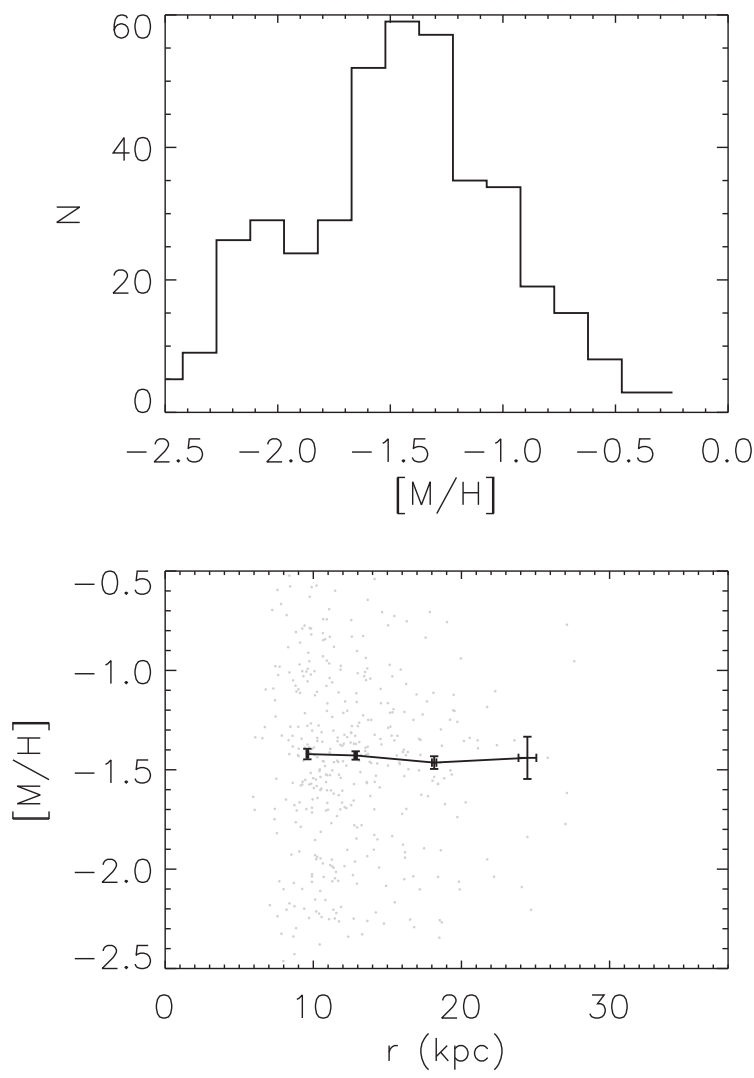

Figure 1. Top panel: MDF derived from the calibrated $[M / H]$ for our sample of 410 halo stars with $|z|>5 \mathrm{kpc}$. Bottom panel: median $[M / \mathrm{H}]$ (calibrated) as a function of the distance from the Galactic Centre, $r$, calculated with distances by the Brazilian Participation Group - see Section 3.3 - (from the peak of their second PDF) for the same sample.

(ii) individual chemical abundances are derived by searching only in the $[\mathrm{Fe} / \mathrm{H}]$ dimension, with the $T_{\text {eff }}$ and $\log g$ fixed at the previously determined values, and fitting isolated spectral windows dominated by features of the element of interest.

ASPCAP includes several improvements, and performs a more refined abundance determination than FA15. For instance, the synthetic grid includes separate $[\mathrm{C} / \mathrm{Fe}],[\mathrm{N} / \mathrm{Fe}]$, and $[\alpha / \mathrm{Fe}]$ dimensions, and the atmospheric models in the synthetic spectra generation are consistent with the variations in $\mathrm{C}$ and the $\alpha$-element abundances. An improved atomic line list is used, and other upgrades (broadening to account for macroturbulent velocity, etc.) are considered (for more details, see García Pérez et al. 2016). Most importantly, the higher S/N (>100) and resolving power $(R \sim 22500)$ of APOGEE spectra allow for an improvement of the accuracy of estimates compared with those obtained from the lower resolution optical spectra. The spectral features resolved in the near-infrared $H$ band also permit the measurement of many more chemical elements. On the other hand, APOGEE was designed to observe mainly the Galactic disc and bulge. For this reason, the survey targeted very few halo stars at distances farther than $30 \mathrm{kpc}$ from the Sun. Therefore, we cannot explore the trends in the most distant regions of the halo investigated in FA15, which included stars with Galactocentric distances beyond $40 \mathrm{kpc}$.

$H$-band stellar spectra generally exhibit weaker lines than optical spectra. With a minimum opacity at the transition between the dominance of continuum $\mathrm{H}^{-}$bound-free and free-free opacity at about $1.6 \mu \mathrm{m}$, in the centre of the $H$ band, photons escape from 
deeper atmospheric layers in the $H$ band than in the optical spectral of late-type stars. Deeper layers are warmer and produce weaker absorption lines, and $H$-band transitions tend to have higher excitation, which makes them weaker as well. Fewer and weaker lines, even though they are less dependent on the choice of microturbulence, mean more limited information in the spectra. In addition, metal-poor atmospheres have higher gas pressure, increasing the role of line damping, and a reduced opacity enhances departures from local thermodynamical equilibrium. These effects may limit the accuracy and precision of the APOGEE abundances for metalpoor stars more than for their solar-metallicity counterparts.

As in FA15, we would like to evaluate how the individual elemental abundances vary with distance from the Galactic Centre and stellar metallicity. In FA15, we took our individual iron abundance measurements $([\mathrm{Fe} / \mathrm{H}])$ as an indicator of the metallicity, $[M / \mathrm{H}]$, in the stars. In the present paper, we also consider this elemental abundance as the primary estimate of stellar metallicity.

The variation of the iron abundance with respect to the solar value is considered in ASPCAP as a dimension of the synthetic library. All the other elements, except $\mathrm{C}, \mathrm{N}$, and the $\alpha$-elements, change in the same proportion as iron with respect to solar abundances. ASPCAP provides two estimates for the iron abundance. On the one hand, an iron abundance measurement $([M / \mathrm{H}])$ is obtained from the fit of the entire available APOGEE spectral range, which includes spectral features from several chemical elements. On the other hand, another estimate $([\mathrm{Fe} / \mathrm{H}])$ is derived by seeking the best match in the $[M / \mathrm{H}]$ dimension, but fitting only spectral windows containing iron lines (García Pérez et al. 2016). Both measurements are expected to be quite close to one another.

A systematic overestimate at low metallicities was detected in Holtzman et al. (2015) for both $[M / \mathrm{H}]$ and $[\mathrm{Fe} / \mathrm{H}]$ by comparing with $[\mathrm{Fe} / \mathrm{H}]$ measurements from the literature. Consequently, they performed an external calibration to $[M / \mathrm{H}]$ (a second-order fit) which corrects for this effect, but this was not applied to $[\mathrm{Fe} / \mathrm{H}]$. Moreover, each individual element was internally calibrated independently from the others to remove abundance trends with effective temperature in open clusters.

In the case of $\mathrm{C}, \mathrm{N}$, and the $\alpha$-elements, ASPCAP calculates their variation over Fe by directly searching within the library. We use these quantities when evaluating $[X / F e]$ for these elements. For the other elements, we calculate $[X / F e]$ ratios from internally calibrated individual chemical abundances (including $[\mathrm{Fe} / \mathrm{H}]$ ). It is not yet clear what might be the cause of the $[\mathrm{Fe} / \mathrm{H}]$ systematic deviation at low metallicities, and other individual abundances may be affected as well. However, ratios in the form $[X / F e]$ from measurements with the same systematic deviation cancel this effect.

We are interested in evaluating differences in the behaviours of individual elemental abundances. The $[M / \mathrm{H}]$ determination is influenced by the contribution of elements other than iron, which can induce deviation from the true iron abundance. Consequently, the $[X / M]$ ratios may not be reliable for our purposes, so we avoid their use in this paper.

Additionally, we are interested in evaluating the chemical trends in different metallicity bins. We choose the calibrated $[M / \mathrm{H}]$ as our indicator of the global metallicity, because it is corrected for the overestimation on the metal-poor side. $[\mathrm{Fe} / \mathrm{H}]$ is unsuitable in this case, because it is still affected by the systematic deviation. Considering it to derive trends with metallicity would place metal-poor stars in higher metallicity bins, and the resulting trends would be distorted. Thus, we use internally calibrated abundance estimates when discussing abundance ratios, but employ the externally calibrated $[M / \mathrm{H}]$ to set our metallicity scale.
Notice that the analysis in Holtzman et al. (2015) revealed hints of 'some issue which may be affecting the reliability of the ASPCAP $[\mathrm{Ti} / \mathrm{H}]$ abundance', and a large scatter in $[\mathrm{Na} / \mathrm{H}]$ and $[\mathrm{V} / \mathrm{H}]$, which also lead one to be aware of the limited precision of these abundance estimates. For these reasons, we cautiously interpret the resulting trends for these elements.

\subsection{Distances}

A number of independent groups have been working on the derivation of distance estimates for APOGEE stars, which we consider in our present analysis; these are described in Santiago et al. (2016), Hayden et al. (2015), and Schultheis et al. (2014).

The derivation of distances for APOGEE giant stars necessarily involves dealing with stars with a very wide range of luminosities, increasing the susceptibility to uncertainties in the stellar evolution models adopted. Nevertheless, comparison across different implementations and with Gaia/Hipparcos parallaxes suggests that no significant systematic errors are present in the distances adopted in this paper.

Distances derived by the SDSS-III Brazilian Participation Group (BPG; Santiago et al. 2016) were computed using the Bayesian methodology explained in Burnett \& Binney (2010), Burnett et al. (2011), and Binney et al. (2014). From the measured spectroscopic parameters coupled with 2MASS photometry, they obtained the posterior distance probability distribution function (PDF) for each star over a grid of PARSEC (Bressan et al. 2012) stellar evolutionary models. Their model prior includes information such as the spatial distribution of stars in our Galaxy and the IMF.

The BPG considered the ASPCAP $[M / \mathrm{H}]$ calibrated values, except for metallicities $[M / \mathrm{H}]>0.0$, because in this regime stars may be 'over'-calibrated, due to the choice of a second-order fit to the data values running away at the edges of its range of validity. They also applied an additional surface gravity calibration with respect to the DR12 $\log g$ values for stars belonging to the red clump. This does not affect our sample because we only consider stars with $\log g<3.5$, which are not members of the red clump. The accuracy of their results was tested with simulations and previous distance estimates for several samples of observations from the literature. The statistical distance uncertainties are at a level of 20 per cent. Although we cannot completely exclude this possibility, there are no strong indications of systematic distance biases towards large distances (low gravities).

Hayden et al. (2015, hereafter H15) derived distances following the same methodology as the BPG. They compared the stellar parameters from ASPCAP with PARSEC isochrones from the Padova-Trieste group (Bressan et al. 2012), considering matches within $3 \sigma$. They then computed the PDF of all distance moduli in the range between the minimum and maximum magnitudes matching the isochrone grid. As for the BPG estimates, the precisions are at a level of 15-20 per cent.

Finally, the methodology followed by Schultheis et al. (2014, hereafter S14) consisted of comparing with Padova isochrones from Marigo et al. (2008), and looking for those which match most closely with the ASPCAP parameters $T_{\text {eff }}, \log g$, and calibrated $[M / \mathrm{H}]$. These authors recognized that not taking into account the $\alpha$ element enhancements and the use of solar-scaled isochrones may introduce errors in their distance estimates. Thus, they estimated the median precisions in their derived distances to be of the order of $\sim 30-40$ per cent. 
From these three sets of distance estimates, we determine distances from the Galactic Centre, $r$, as follows:

$r=\sqrt{d^{2}+\mathrm{R}_{\odot}^{2}-2 d \mathrm{R}_{\odot} \cos b \cos l}$

and the distance from the Galactic plane, $z$,

$z=d \sin b$,

where $b$ and $l$ are the Galactic coordinates, provided in the APOGEE data files, and $\mathrm{R}_{\odot}=8.0 \mathrm{kpc}$, given by Ghez et al. (2008).

\subsection{Evaluation of the chemical trends}

We now consider the variation of individual chemical abundances, as a function of distance from the Galactic Centre, for each of the 15 elements determined by ASPCAP. Our sample covers the range $5 \lesssim r \lesssim 30 \mathrm{kpc}$. We inferred their trends by calculating the median $[X / \mathrm{H}]$ in bins of $\Delta r=5 \mathrm{kpc}$ or wider, assuring a minimum of 100 stars per bin. Figs 1 (bottom panel) and 2 show the resulting trends.

We are also interested in examining how the elemental abundance-to-iron ratios vary with $r$ and $[M / \mathrm{H}]$. For this purpose, we split our sample into three metallicity bins $(-2.5<[M / \mathrm{H}]<$ $-1.8,-1.8<[M / \mathrm{H}]<-1.1$, and $-1.1<[M / \mathrm{H}]<0.0)$, and calculate the median ratios for stars at $r<10,10<r<15$, and $r>15 \mathrm{kpc}$, in each one of the three metallicity ranges. The choice of these bins satisfies our aim to calculate the median ratios from the largest possible data sets, in order to infer the chemical trends as accurately as possible. Figs 3 and 4 show the resulting median $[X / F e]$ ratios, as a function of $r$ and $[M / \mathrm{H}]$, respectively, evaluated separately in the corresponding metallicity and distance bins.

We indicate with error bars the median absolute deviation (MAD) divided by the square root of the number of points from which we derive each median abundance (we assume that the uncertainties follow a Gaussian distribution). The abundance dispersion known for the halo is $\sim 0.5$ dex (Allende Prieto et al. 2014). The bulk of the $[X / H]$ and $[X / F e]$ uncertainties are $\sim 0.1$ with few exceptions $([\mathrm{Na} / \mathrm{H}]$ and $[\mathrm{V} / \mathrm{H}])$, and in no cases exceed $0.3 \mathrm{dex}$, on average, in each bin. Consequently, our sample should be dominated by the natural halo abundance dispersion. However, we also estimate the weighted mean with the uncertainties provided in the APOGEE data base, in order to test that the resulting trends are not significantly distorted due to the abundance errors.

In order to quantify the variation, Table 1 shows the difference between each median $[X / \mathrm{Fe}]$ ratio with the nearest stars median $[X / \mathrm{Fe}], r<10 \mathrm{kpc}$, for each range of $[M / \mathrm{H}]$ considered and with the lowest metallicity median, $-2.5<[M / \mathrm{H}]<-1.8$, for each range of $r$. When the difference is significant [as demonstrated by application of a Kolmogorov-Smirnov (K-S) test - see Section 4.4], it is indicated in italics.

Finally, we verify whether the resulting trends are consistent when taking into account the three sets of available distance estimates. For this purpose, we analyse whether the variance of the median $[X / F e]$ ratios, calculated from the distance estimates by the several groups described in Section 4.4, follows the same trends inferred from an individual set of estimates.

\section{RESULTS}

\section{$4.1[X / H]$ versus $r$}

We evaluate the median $[\mathrm{Fe} / \mathrm{H}]$ values, as a function of $r$, using distances calculated by BPG, H15, and S14. All produce fairly similar results, but we choose to employ the BPG estimates derived from the peak of the second PDF in their analysis (BPG2p - see Santiago et al. 2016). The reason is that it has the least amount of scatter in the $[M / \mathrm{H}]$ versus $r$ relation, and showed little signs of a gradient, which is in agreement with that observed by FA15.

Considering the $[M / \mathrm{H}]$ values externally calibrated with $[\mathrm{Fe} / \mathrm{H}]$ abundances from the literature, the resulting median values (bottom panel in Fig. 1) are around $\sim-1.5$, which is consistent with the previous works. As mentioned above in Section 3.1, we have derived the MDF for our sample from the calibrated $[M / \mathrm{H}]$, shown in the top panel of Fig. 1. The peak of the distribution is around $[M / \mathrm{H}]$ $\sim-1.5$. In addition, a second peak around -2.1 is observed. This is very close to the median metallicity value associated with the outer-halo region (Carollo et al. 2010; Allende Prieto et al. 2014; FA15).

The trend of the median $[X / \mathrm{H}]$ ratios with distance from the Galactic Centre, shown in Fig. 2, exhibits constant or decreasing trends. Those inferred for the $\alpha$-elements are fairly constant, except $[\mathrm{Mg} / \mathrm{H}]$ and $[\mathrm{Ti} / \mathrm{H}]$, which show a significant decrease, $\sim 0.1 \mathrm{dex}$, from $r<10$ to $r>15 \mathrm{kpc}$. [C/H] also exhibits a significant variation, the largest among all the elements evaluated, decreasing by $\sim 0.2 \mathrm{dex}$ from $r<10$ to $10<r<15 \mathrm{kpc}$.

It is important to recall, however, that the abundances of $[\mathrm{C} / \mathrm{H}]$ and $[\mathrm{N} / \mathrm{H}]$ can be altered due to mixing events in cool red giants, the dominant spectral class of our sample. Thus, these elements are not reliable indicators of the ISM chemistry from which these stars formed. Although we provide their resulting median values and trends, we will not comment on them because we are interested in those abundances which provide information of the previous stellar populations.

The $[\mathrm{Mn} / \mathrm{H}]$ abundance exhibits a decreasing trend, contrary to the other iron-peak $[\mathrm{Ni} / \mathrm{H}]$, which does not show significant variation. The elements $[\mathrm{Na} / \mathrm{H}]$ and $[\mathrm{Al} / \mathrm{H}]$ both exhibit decreasing trends, although $[\mathrm{Na} / \mathrm{H}]$ has a larger variance with distance ( $\sim 0.4 \mathrm{dex})$. [Al/H] decreases $\sim 0.1$ dex from $r<10 \mathrm{kpc}$ to $10<$ $r<15 \mathrm{kpc}$. Both elements are produced by massive stars and LIMS; however, the production and ejection efficiencies for each element are different. Finally, the $[\mathrm{K} / \mathrm{H}]$ and $[\mathrm{V} / \mathrm{H}]$ both show constant trends.

We also evaluate $[X / H]$ trends with $r$, splitting the sample into bins of $[M / \mathrm{H}]$. As expected, $[X / \mathrm{H}]$ is higher as $[M / \mathrm{H}]$ increases. Overall, the elemental abundances exhibit similar variations with $r$ and $[M / H]$. The most metal-rich stars exhibit the largest variation with $r$, with higher median values for stars in the inner-halo region compared to those in outer-halo region, excepting $[\mathrm{Si} / \mathrm{H}]$ and $[\mathrm{Ti} / \mathrm{H}]$ among the $\alpha$-elements, and $[\mathrm{N} / \mathrm{H}],[\mathrm{Ni} / \mathrm{H}]$, and $[\mathrm{V} / \mathrm{H}]$, which remain constant.

\section{$4.2[X / F e]$ versus $r$}

Inspection of the variation of $[X / \mathrm{Fe}]$ with $r$ reveals that the chemical trends depend on the metallicity range considered, as seen in Fig. 3. For the $\alpha$-elements, metal-poor stars have enhanced $[X / \mathrm{Fe}]$ across all $r$. The median ratios decrease as the metallicity increases. The most metal-rich stars show the largest variation with $r$, decreasing farther from the Galactic Centre. This decreasing trend tends to flatten towards lower $[M / \mathrm{H}]$, although $[\mathrm{Ca} / \mathrm{Fe}]$ shows a significant fluctuation of 0.07 dex with $r$ at $-2.5<[M / \mathrm{H}]<-1.8$. [Ti/Fe] decreases 0.14 dex in this metallicity bin.

At $-1.1<[M / \mathrm{H}]<0.0,[\mathrm{O} / \mathrm{Fe}],[\mathrm{Mg} / \mathrm{Fe}]$, and $[\mathrm{S} / \mathrm{Fe}]$ exhibit the largest variation, $\sim 0.2 \mathrm{dex}$, with $r$. The $[\mathrm{Si} / \mathrm{Fe}],[\mathrm{Ca} / \mathrm{Fe}]$, and [Ti/Fe] ratios decrease by almost 0.1 dex between the inner and 

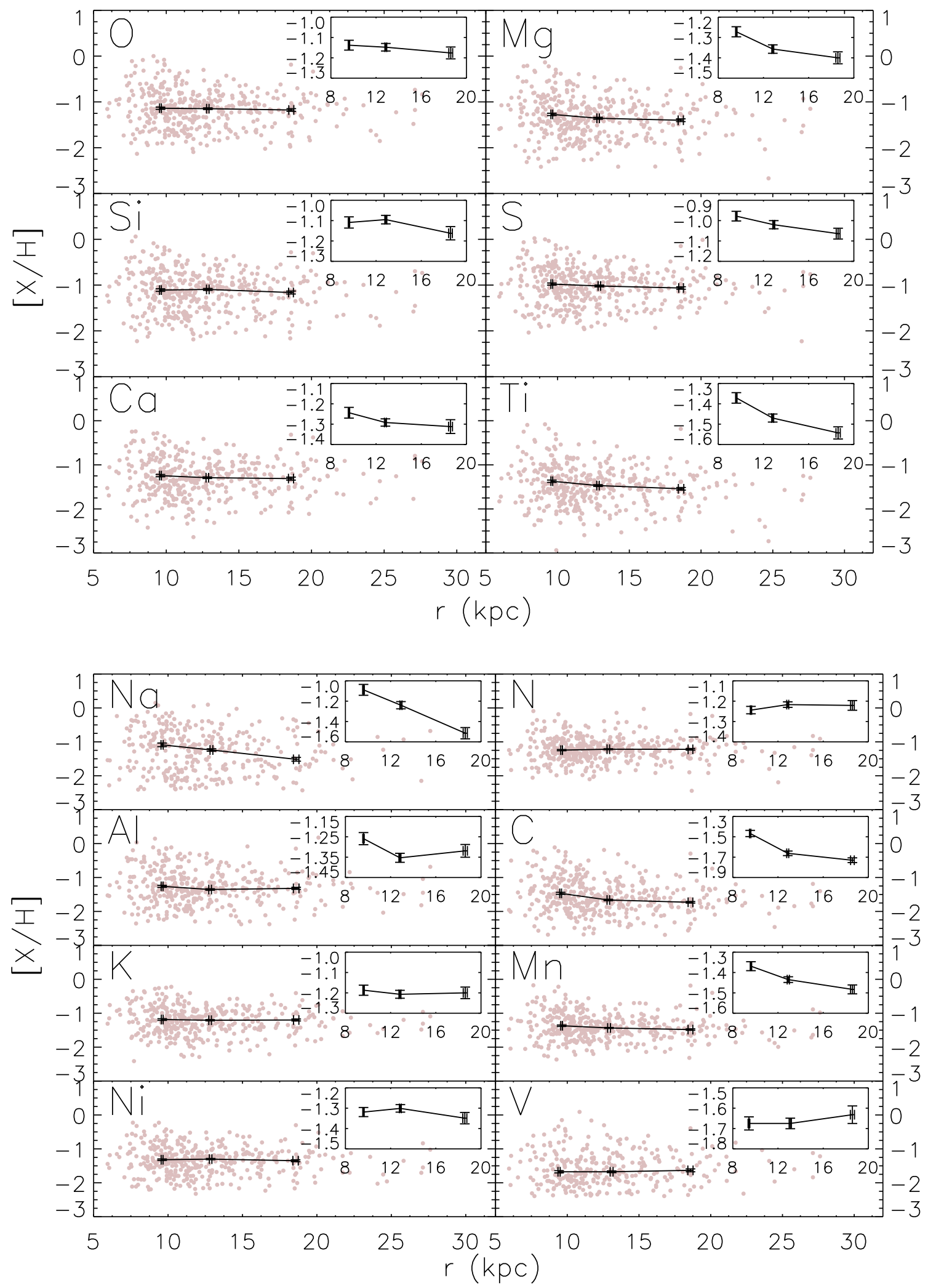

Figure 2. $[X / H]$ median values as a function of the distance from the Galactic Centre, $r$. The top panels correspond to the $\alpha$-elements and the bottom panels to the other analysed elements. The median values with axes covering narrower ranges which emphasize their behaviour are shown as insets at the upper right in each panel. 

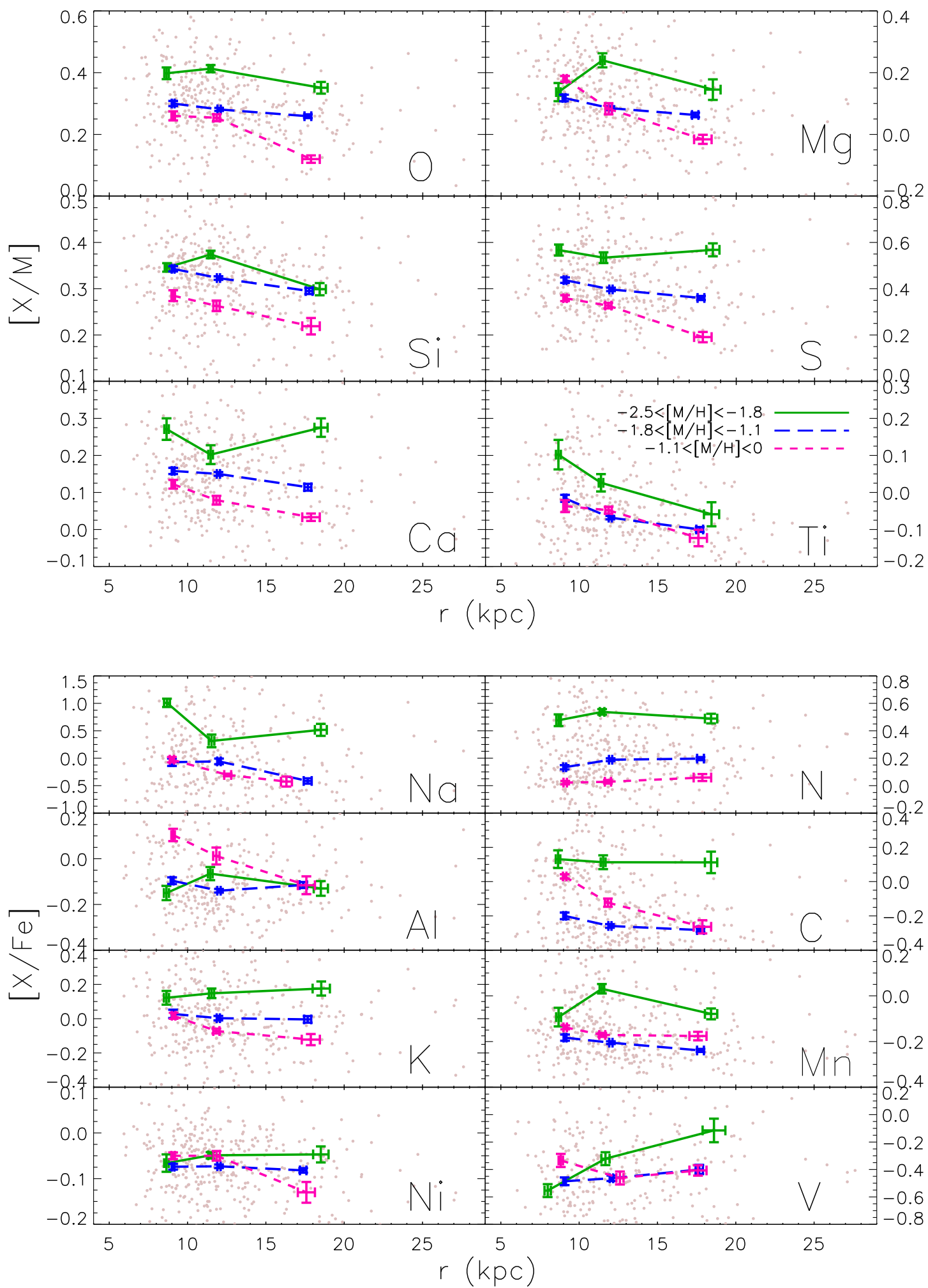

Figure 3. $[X / \mathrm{Fe}]$ median values as a function of $r$. The trends are inferred by splitting the sample into the three $[M / \mathrm{H}]$ bins shown in the legend of the lower-right panel in the top set of plots.

outer regions. As was found by $\mathrm{FA} 15,[\mathrm{Ca} / \mathrm{Fe}]$ exhibits a larger dependence with metallicity than $[\mathrm{Mg} / \mathrm{Fe}]$. The decreasing trends of $[\mathrm{Ca} / \mathrm{Fe}]$ with $r$ in each of the metallicity bins analysed are consistent with the FA15 results, although with an offset in the median values. In contrast, the increasing trends observed in FA15 for $[\mathrm{Mg} / \mathrm{Fe}]$ at
$[M / H]>-1.1$ are not confirmed in this work, where we find that the median ratio decreases.

The iron-peak elements $\mathrm{Ni}$ and $\mathrm{Mn}$ do not exhibit ratios which strongly vary with $r$. Metal-poor stars show enhanced [Mn/Fe], the largest of $\sim 0.13 \mathrm{dex}$ at $10<r<15 \mathrm{kpc}$. Noticeably, $[\mathrm{Ni} / \mathrm{Fe}]$ tends 

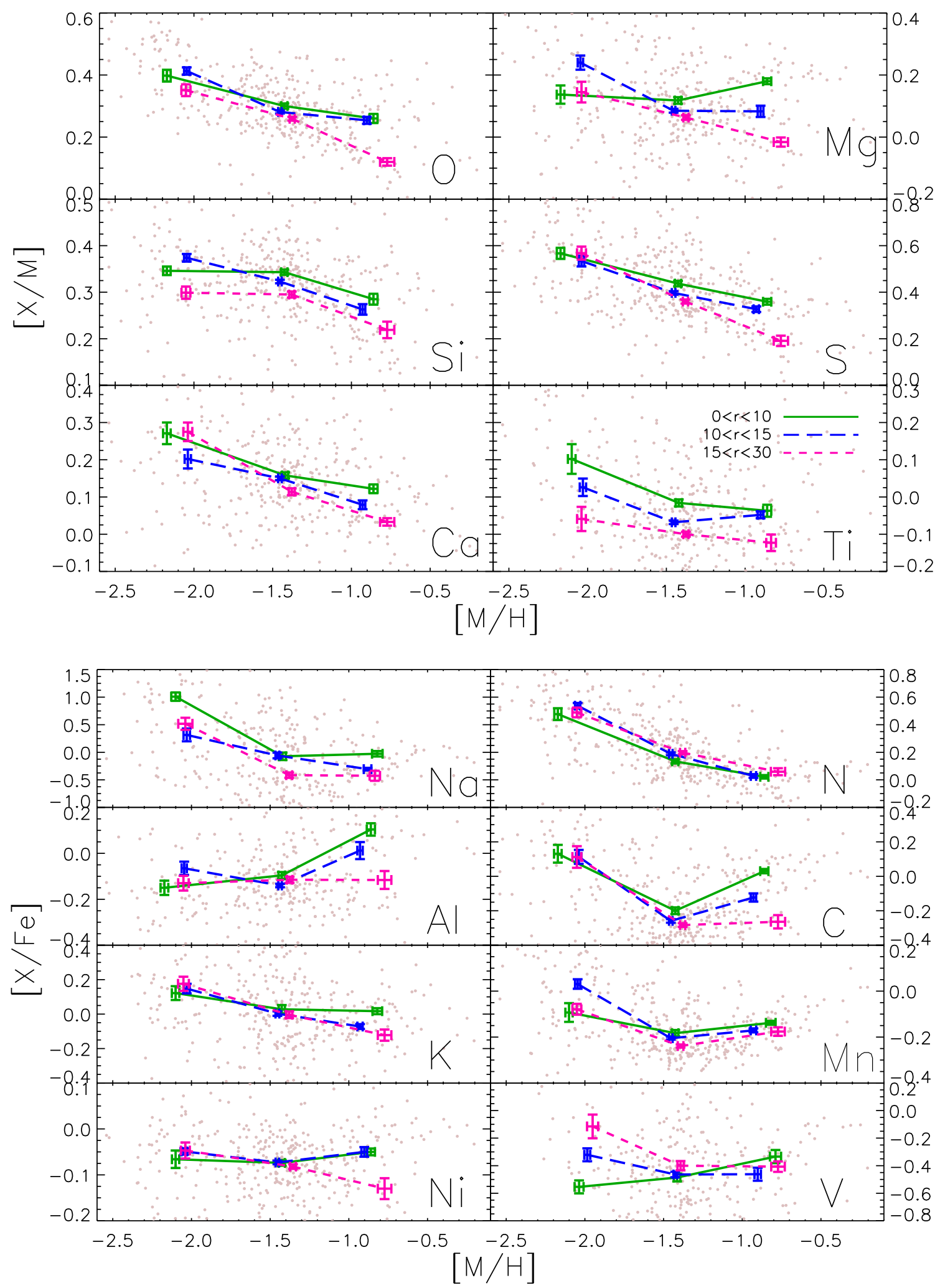

Figure 4. $[X / \mathrm{Fe}]$ median values as a function of $[M / \mathrm{H}]$, splitting the sample into three $r$ bins: $r<10 \mathrm{kpc}, 10<r<15 \mathrm{kpc}$, and $r>15 \mathrm{kpc}$.

to decrease $(\sim 0.08 \mathrm{dex})$ with distance for stars in the most metalrich bin. This pattern is the same observed for the $\alpha$-elements, in agreement with previous findings (Nissen \& Schuster 2010, 2011; Yamada et al. 2013; Hawkins et al. 2015), although they detected the pattern from the analysis of $[X / F e]$ as a function of metallicity.
$[\mathrm{Na} / \mathrm{Fe}]$ and $[\mathrm{Al} / \mathrm{Fe}]$ do not follow the same trends with $r$ and $[M / \mathrm{H}]$. The $[\mathrm{Na} / \mathrm{Fe}]$ ratio decreases with $r$, and exhibits higher median values as metallicity decreases. In contrast, $[\mathrm{Al} / \mathrm{Fe}]$ exhibits a different pattern depending on the distance bin and metallicity range considered. In the inner regions, $r<10 \mathrm{kpc}$, $[\mathrm{Al} / \mathrm{Fe}]$ is higher 
Table 1. Median $[X / \mathrm{Fe}]$ and $r$ with their corresponding MAD, evaluated in the three $[M / \mathrm{H}]$ and $r$ bins, and the difference between each median and that corresponding to the lowest $r$ bin over each $[M / \mathrm{H}]$ range and to the lowest $[M / \mathrm{H}]$ bin over each $r$ range. The significant differences indicated by the $\mathrm{K}-\mathrm{S}$ test are in italics, followed by the level of significance (los).

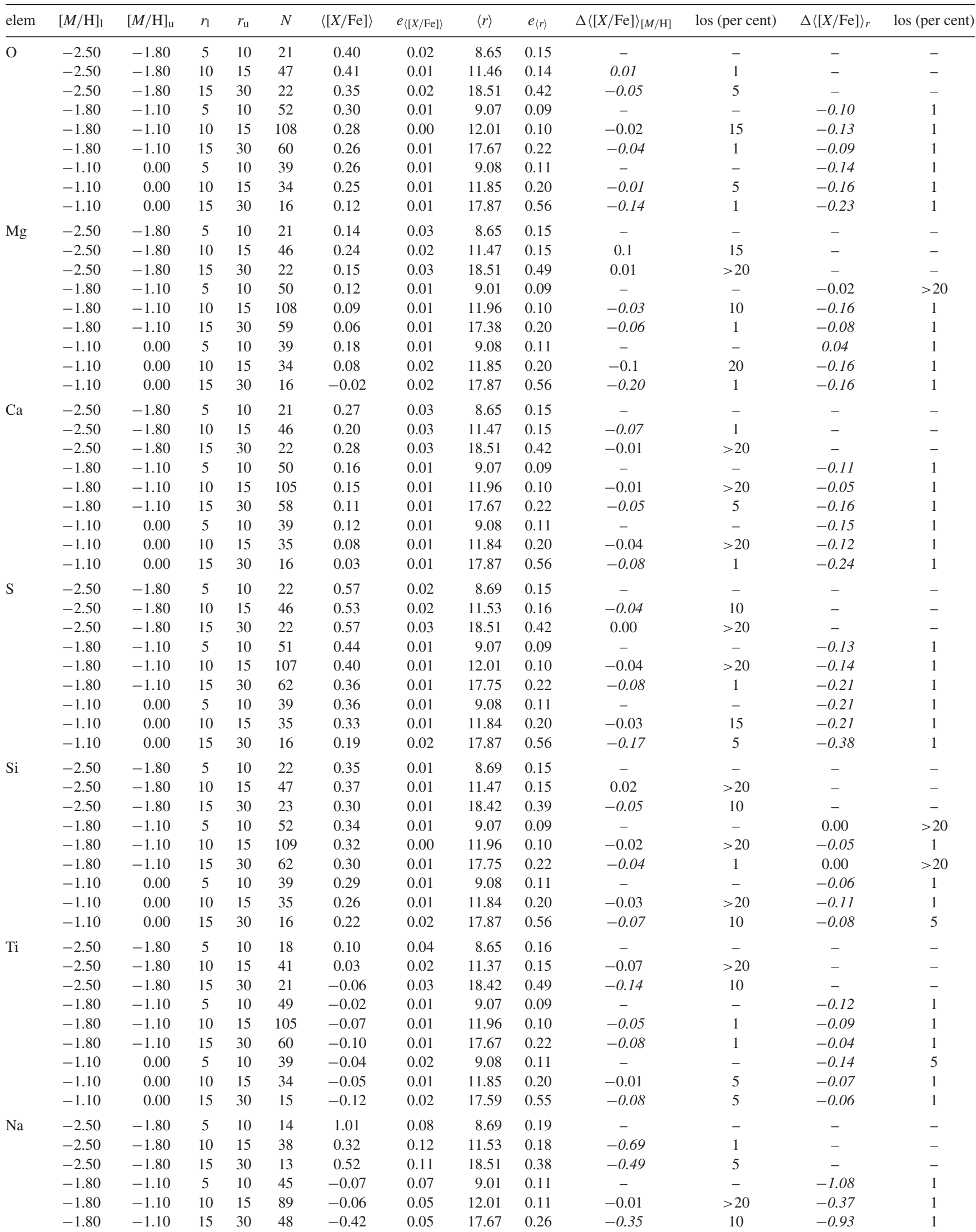


Table 1 - continued

\begin{tabular}{|c|c|c|c|c|c|c|c|c|c|c|c|c|c|}
\hline elem & {$[M / \mathrm{H}]_{1}$} & {$[M / \mathrm{H}]_{\mathrm{u}}$} & $r_{1}$ & $r_{\mathrm{u}}$ & $N$ & $\langle[X / \mathrm{Fe}]\rangle$ & $e_{\langle[X / \mathrm{Fe}]\rangle}$ & $\langle r\rangle$ & $e_{\langle r\rangle}$ & $\Delta\langle[X / \mathrm{Fe}]\rangle_{[M / \mathrm{H}]}$ & los (per cent) & $\Delta\langle[X / \mathrm{Fe}]\rangle_{r}$ & los (per cent) \\
\hline & -1.10 & 0.00 & 10 & 15 & 28 & -0.31 & 0.02 & 12.56 & 0.22 & -0.28 & 1 & -0.62 & 1 \\
\hline & -1.10 & 0.00 & 15 & 30 & 13 & -0.43 & 0.09 & 16.31 & 0.35 & -0.40 & 5 & -0.94 & 1 \\
\hline \multirow[t]{7}{*}{ N } & -2.50 & -1.80 & 5 & 10 & 23 & 0.48 & 0.04 & 8.69 & 0.15 & - & - & - & - \\
\hline & -2.50 & -1.80 & 10 & 15 & 47 & 0.54 & 0.02 & 11.47 & 0.15 & 0.06 & $>20$ & - & - \\
\hline & -2.50 & -1.80 & 15 & 30 & 23 & 0.49 & 0.04 & 18.42 & 0.39 & 0.01 & $>20$ & - & - \\
\hline & -1.80 & -1.10 & 15 & 30 & 62 & 0.20 & 0.01 & 17.75 & 0.22 & 0.07 & 5 & -0.29 & 1 \\
\hline & -1.10 & 0.00 & 5 & 10 & 40 & 0.02 & 0.01 & 9.11 & 0.11 & - & - & -0.46 & 1 \\
\hline & -1.10 & 0.00 & 10 & 15 & 35 & 0.03 & 0.01 & 11.84 & 0.20 & 0.01 & $>20$ & -0.51 & 1 \\
\hline & -1.10 & 0.00 & 15 & 30 & 16 & 0.06 & 0.03 & 17.87 & 0.56 & 0.04 & $>20$ & -0.43 & 1 \\
\hline \multirow[t]{5}{*}{$\mathrm{Al}$} & -2.50 & -1.80 & 5 & 10 & 20 & -0.15 & 0.03 & 8.65 & 0.16 & - & - & - & - \\
\hline & -2.50 & -1.80 & 10 & 15 & 45 & -0.07 & 0.03 & 11.45 & 0.15 & 0.08 & 1 & - & - \\
\hline & -1.10 & 0.00 & 5 & 10 & 39 & 0.10 & 0.03 & 9.08 & 0.11 & - & - & 0.25 & 1 \\
\hline & -1.10 & 0.00 & 10 & 15 & 34 & 0.01 & 0.04 & 11.84 & 0.20 & -0.09 & 5 & 0.08 & 5 \\
\hline & -1.10 & 0.00 & 15 & 30 & 15 & -0.12 & 0.04 & 17.59 & 0.55 & -0.22 & 10 & 0.01 & 5 \\
\hline \multirow[t]{9}{*}{$\mathrm{C}$} & -2.50 & -1.80 & 5 & 10 & 21 & 0.13 & 0.05 & 8.65 & 0.14 & - & - & - & - \\
\hline & -2.50 & -1.80 & 10 & 15 & 46 & 0.11 & 0.04 & 11.53 & 0.16 & -0.02 & 5 & - & - \\
\hline & -2.50 & -1.80 & 15 & 30 & 23 & 0.11 & 0.06 & 18.42 & 0.39 & -0.02 & $>20$ & - & - \\
\hline & -1.80 & -1.10 & 5 & 10 & 52 & -0.20 & 0.02 & 9.07 & 0.09 & - & - & -0.33 & 1 \\
\hline & -1.80 & -1.10 & 10 & 15 & 109 & -0.26 & 0.01 & 11.96 & 0.10 & -0.06 & 5 & -0.37 & 1 \\
\hline & -1.80 & -1.10 & 15 & 30 & 62 & -0.28 & 0.01 & 17.75 & 0.22 & -0.08 & 1 & -0.40 & 1 \\
\hline & -1.10 & 0.00 & 5 & 10 & 39 & 0.03 & 0.01 & 9.08 & 0.11 & - & - & -0.10 & 1 \\
\hline & -1.10 & 0.00 & 10 & 15 & 35 & -0.12 & 0.03 & 11.84 & 0.20 & -0.15 & 1 & -0.24 & 1 \\
\hline & -1.10 & 0.00 & 15 & 30 & 16 & -0.26 & 0.04 & 17.87 & 0.56 & -0.29 & 1 & -0.38 & 1 \\
\hline K & -1.10 & 0.00 & 15 & 30 & 15 & -0.12 & 0.03 & 17.87 & 0.58 & -0.14 & 5 & -0.30 & 1 \\
\hline \multirow[t]{9}{*}{$\mathrm{Mn}$} & -2.50 & -1.80 & 5 & 10 & 20 & -0.09 & 0.04 & 8.69 & 0.16 & - & - & - & - \\
\hline & -2.50 & -1.80 & 10 & 15 & 45 & 0.03 & 0.02 & 11.45 & 0.15 & 0.13 & 1 & - & - \\
\hline & -2.50 & -1.80 & 15 & 30 & 23 & -0.08 & 0.02 & 18.42 & 0.39 & 0.021 & $>20$ & - & - \\
\hline & -1.80 & -1.10 & 5 & 10 & 50 & -0.18 & 0.01 & 9.10 & 0.09 & - & - & -0.09 & 1 \\
\hline & -1.80 & -1.10 & 10 & 15 & 105 & -0.21 & 0.01 & 12.02 & 0.11 & 0.01 & 5 & -0.24 & 1 \\
\hline & -1.80 & -1.10 & 15 & 30 & 61 & -0.24 & 0.01 & 17.75 & 0.22 & -0.01 & 1 & -0.16 & 1 \\
\hline & -1.10 & 0.00 & 5 & 10 & 38 & -0.14 & 0.01 & 9.11 & 0.11 & - & - & -0.05 & 1 \\
\hline & -1.10 & 0.00 & 10 & 15 & 33 & -0.17 & 0.01 & 11.48 & 0.19 & -0.01 & $>20$ & -0.20 & 1 \\
\hline & -1.10 & 0.00 & 15 & 30 & 15 & -0.18 & 0.02 & 17.59 & 0.55 & -0.02 & $>20$ & -0.10 & 10 \\
\hline \multirow[t]{9}{*}{$\mathrm{Ni}$} & -2.50 & -1.80 & 5 & 10 & 20 & -0.07 & 0.02 & 8.65 & 0.15 & - & - & - & - \\
\hline & -2.50 & -1.80 & 10 & 15 & 46 & -0.05 & 0.01 & 11.47 & 0.15 & 0.02 & $>20$ & - & - \\
\hline & -2.50 & -1.80 & 15 & 30 & 22 & -0.05 & 0.02 & 18.51 & 0.49 & 0.02 & $>20$ & - & - \\
\hline & -1.80 & -1.10 & 5 & 10 & 51 & -0.07 & 0.01 & 9.07 & 0.09 & - & - & -0.01 & 1 \\
\hline & -1.80 & -1.10 & 10 & 15 & 108 & -0.07 & 0.00 & 12.01 & 0.10 & 0.00 & $>20$ & -0.02 & 1 \\
\hline & -1.80 & -1.10 & 15 & 30 & 58 & -0.08 & 0.01 & 17.38 & 0.21 & -0.01 & $>20$ & -0.04 & 5 \\
\hline & -1.10 & 0.00 & 5 & 10 & 39 & -0.05 & 0.01 & 9.08 & 0.11 & - & - & 0.02 & $>20$ \\
\hline & -1.10 & 0.00 & 10 & 15 & 34 & -0.05 & 0.01 & 11.85 & 0.20 & 0.00 & $>20$ & 0.00 & $>20$ \\
\hline & -1.10 & 0.00 & 15 & 30 & 15 & -0.13 & 0.02 & 17.59 & 0.55 & -0.08 & 5 & -0.08 & $>20$ \\
\hline \multirow[t]{4}{*}{ V } & -2.50 & -1.80 & 5 & 10 & 5 & -0.55 & 0.05 & 7.99 & 0.20 & - & - & - & - \\
\hline & -2.50 & -1.80 & 10 & 15 & 22 & -0.32 & 0.05 & 11.68 & 0.26 & 0.23 & 1 & - & - \\
\hline & -2.50 & -1.80 & 15 & 30 & 11 & -0.12 & 0.09 & 18.61 & 0.73 & 0.43 & 1 & - & - \\
\hline & -1.80 & -1.10 & 5 & 10 & 41 & -0.49 & 0.03 & 9.07 & 0.08 & - & - & 0.07 & 1 \\
\hline
\end{tabular}


Table 1 - continued

\begin{tabular}{cccccccccccccc}
\hline elem & {$[M / \mathrm{H}]_{\mathrm{l}}$} & \multicolumn{1}{l}{$[M / \mathrm{H}]_{\mathrm{u}}$} & $r_{\mathrm{l}}$ & $r_{\mathrm{u}}$ & $N$ & $\langle[X / \mathrm{Fe}]\rangle$ & $e_{\langle[X / \mathrm{Fe}]\rangle}$ & $\langle r\rangle$ & $e_{\langle r\rangle}$ & $\Delta\langle[X / \mathrm{Fe}]\rangle_{[M / \mathrm{H}]}$ & $\operatorname{los}($ per cent) & $\Delta\langle[X / \mathrm{Fe}]\rangle_{r}$ & $\operatorname{los}(\mathrm{per} \operatorname{cent})$ \\
\hline-1.80 & -1.10 & 10 & 15 & 89 & -0.46 & 0.02 & 12.06 & 0.11 & 0.03 & $>20$ & -0.14 \\
-1.80 & -1.10 & 15 & 30 & 52 & -0.40 & 0.03 & 17.67 & 0.23 & 0.09 & 1 & 10 & -0.29 \\
-1.10 & 0.00 & 5 & 10 & 33 & -0.33 & 0.05 & 8.83 & 0.14 & - & - & 0.22 \\
-1.10 & 0.00 & 10 & 15 & 30 & -0.46 & 0.05 & 12.60 & 0.23 & -0.13 & $>20$ & -0.14 & 1 \\
-1.10 & 0.00 & 15 & 30 & 15 & -0.41 & 0.04 & 17.59 & 0.55 & -0.08 & $>20$ & -0.29 & 1 \\
\hline
\end{tabular}

as metallicity increases. This ratio decreases with $r$ for the most metal-rich stars, while it tends to increase for the most metal-poor stars. As a consequence, stars in the outer region have $[\mathrm{Al} / \mathrm{Fe}]$ which does not depend so significantly on metallicity than for stars in the inner region. The theoretical $\mathrm{Na}$ and $\mathrm{Al}$ yields predict similar $[X / \mathrm{Fe}]$ versus $[\mathrm{Fe} / \mathrm{H}]$ behaviours. However, observations in the solar neighbourhood do not completely follow the theoretical predictions (Côté et al. 2016). Our analysis also reveals a disagreement in $[\mathrm{Na} / \mathrm{Fe}]$ and $[\mathrm{Al} / \mathrm{Fe}]$ trends with $r$ and $[M / \mathrm{H}]$.

The ratio $[\mathrm{K} / \mathrm{Fe}]$ also tends to decrease with $r$ at $[M / \mathrm{H}] \sim-1.1$, but stars at $[M / \mathrm{H}]<-1.1$ exhibit constant trends. The difference in $[\mathrm{K} / \mathrm{Fe}]$ with $[M / \mathrm{H}]$ is higher for stars in the outer region. [V/Fe] increases with $r$ in the most metal-poor stars, and tends to flatten as $[M / \mathrm{H}]$ increases, although no well-defined trends with metallicity are observed. The chemical analysis of $\mathrm{V}$ should be taken with caution, however, because its measurement is less reliable (V is determined exclusively from very weak spectral features - see Holtzman et al. 2015)

\section{$4.3[X / \mathrm{Fe}]$ versus $[M / \mathrm{H}]$}

The resulting curves of the median $[X / \mathrm{Fe}]$ values, calculated as a function of $[M / \mathrm{H}]$, are shown in Fig. 4. Overall, $\alpha$-elements show decreasing trends with $[M / \mathrm{H}]$, with few exceptions. Stars at $r<$ $10 \mathrm{kpc}$ exhibit a decrease larger than $0.1 \mathrm{dex}$ in $[X / \mathrm{Fe}]$ towards higher $[M / \mathrm{H}]$, except for $[\mathrm{Mg} / \mathrm{Fe}]$ and $[\mathrm{Si} / \mathrm{Fe}]$. These abundances remain constant and slightly vary at $[M / \mathrm{H}] \sim 1.1$, increasing 0.04 dex and decreasing 0.06 dex, respectively.

As $r$ increases, the trends become steeper (except for [Ti/Fe], which tends to flatten). The most distant stars show decreasing variations $>0.1 \mathrm{dex}$ for $[\mathrm{Mg} / \mathrm{Fe}],>0.2$ dex for $[\mathrm{O} / \mathrm{Fe}]$ and $[\mathrm{Ca} / \mathrm{Fe}]$, and $>0.3$ dex for $[\mathrm{S} / \mathrm{Fe}]$. $[\mathrm{Si} / \mathrm{Fe}]$ and $[\mathrm{Ti} / \mathrm{Fe}]$ also decrease with $[M / \mathrm{H}]$, although less $(<0.1 \mathrm{dex})$. Fig. 4 clearly shows the spread in $[\alpha / M]$ for stars at $[M / \mathrm{H}]>-1.1$ as a function of distance from the Galactic Centre described in the previous section. This spread, $\geq 0.1 \mathrm{dex}$, is similar to the differences observed by Nissen \& Schuster (2010) for $[\alpha / \mathrm{Fe}]$ as a function of $[\mathrm{Fe} / \mathrm{H}]$.

Based on the K-S test, no significant variations larger than 0.1 dex are detected in $[\mathrm{Ni} / \mathrm{Fe}]$ with $[\mathrm{M} / \mathrm{H}]$. However, Fig. 2 reveals lower $[\mathrm{Ni} / \mathrm{Fe}]$ as $r$ increases at $[M / \mathrm{H}] \sim-1.1$. The $[\mathrm{Mn} / \mathrm{Fe}]$ ratio decreases with $[M / \mathrm{H}]$ from the most metal-poor stars up to $[M / \mathrm{H}] \sim-1.5$, and increases slightly towards higher metallicities. The increasing trend on the higher metallicity side is independent of distance; all the stars in the sample have similar median $[\mathrm{Mn} / \mathrm{Fe}]$ ratios. In contrast, the metal-poor tail suggests enhanced $[\mathrm{Mn} / \mathrm{Fe}]$ ratios for more distant stars.

There is an overall decrease of $[\mathrm{Na} / \mathrm{Fe}]$ with $[M / \mathrm{H}]$. The $[\mathrm{Na} / \mathrm{Fe}]$ ratio exhibits the largest variation, but also a large scatter, likely due to the difficulty of measuring Na from the APOGEE spectra (Holtzman et al. 2015). The variation of $[\mathrm{Al} / \mathrm{Fe}]$ with $[M / \mathrm{H}]$ clearly depends on the distance bin considered. The nearest stars exhibit an increasing trend, with variations of $\sim 0.25$ dex between stars with
$[M / \mathrm{H}]<-1.8$ and $[M / \mathrm{H}]>-1.1$. This trend tends to flatten with $r$. The $[\mathrm{Al} / \mathrm{Fe}]$ ratio for stars at $r>15 \mathrm{kpc}$ is nearly constant. Thus, for $[M / \mathrm{H}]>-1.1$, the median $[\mathrm{Al} / \mathrm{Fe}]$ decreases with $r$. The metal-poor stars suggest an opposite trend with $r$.

The median $[\mathrm{K} / \mathrm{Fe}]$ ratios also reflect a different enrichment pattern which depends on Galactocentric distance. Trends are steeper as $r$ increases, with enhanced ratios in the metal-poor tail and lower values towards the highest metallicities which we consider. At $[M / \mathrm{H}] \lesssim-1.5$, there is no significant difference in the median ratios calculated for the three distance bins.

We find an increasing trend in $[\mathrm{V} / \mathrm{Fe}]$ with distance for stars at $r<10 \mathrm{kpc}$, flattening as $r$ increases. Our resulting ratios have lower median $[\mathrm{V} / \mathrm{Fe}]$ ratios for all the stars in our sample. Overall, distant stars have higher [V/Fe], and the trends for the three $r$ bins merge for stars with $[M / \mathrm{H}]>-1.1$. However, as mentioned above, estimates of the $\mathrm{V}$ abundance are less reliable due to its weak features in APOGEE spectra.

\subsection{Validation of trends}

In order to check whether the resulting trends reported above depend on the chosen distance estimates, we calculate the median abundance ratios for the six sets of distances available for DR12 APOGEE data. We first calculate the median and its variance for each of the six median abundance ratios in the corresponding $r$ and $[\mathrm{Fe} / \mathrm{H}]$ bins, and then evaluate how these variances change with $r$ and $[M / \mathrm{H}]$. Overall, these curves confirm the previous inferred trends. Thus, the different distance estimates lead to the same qualitative trends, although the particular median values differ slightly depending on the set of distances considered.

As an additional check, we carried out the previous evaluations by considering the mean ratios weighted with the measurement errors - the resulting trends are qualitatively similar to the median ratio curves. We also performed a K-S test in order to verify if the observed differences between our median ratios over bins in $r$ and $[M / \mathrm{H}]$ are statistically significant. We proceed by calculating the cumulative distribution function (CDF) for each bin in $r$ and $[M / H]$ evaluated, and the maximum difference between each CDF and the CDF corresponding to the lowest $r$ bin over each $[M / \mathrm{H}]$ range (and to the lowest $[M / \mathrm{H}]$ bin over each $r$ range), then compare with the critical values of the K-S statistic. In order to consider a difference significant, we demand that it cannot be rejected at higher than the 10 per cent level. We perform the test to evaluate variations of $[X / \mathrm{H}]$ versus $r$ and $[X / \mathrm{Fe}]$ versus $r$ and $[M / \mathrm{H}]$. The last four columns in Table 1 show the resulting variations in $[X / \mathrm{Fe}]$ as a function of $r$ and $[M / \mathrm{H}]$ and the level of significance obtained for them; those with a significant difference are indicated in italics. In the previous sections, we have only described significant variations after applying this test.

Notice that we derive $[X / \mathrm{Fe}]$ (except in the case of $\mathrm{C}, \mathrm{N}$, and $\alpha$ elements) from $[\mathrm{Fe} / \mathrm{H}]$ measurements, which exhibits a systematic deviation comparing with literature values. We do not know whether 
$[X / \mathrm{H}]$ is affected by the same systematic deviation. If this is the case, the deviation would be absent in the resulting $[X / \mathrm{Fe}]$. In contrast, the ratios would be systematically underestimated towards lower metallicities. In most of the cases, the observed $[X / F e]$ trends with metallicity are the opposite. The slopes would be higher if $[X / \mathrm{Fe}]$ were underestimated.

Uncertainties in the stellar parameters could lead to systematic errors in the chemical abundances and thus distortions in the inferred chemical trends. Mészáros et al. (2015) investigated about the deviations in $[\mathrm{Fe} / \mathrm{H}],[\mathrm{C} / \mathrm{Fe}],[\mathrm{N} / \mathrm{Fe}]$, and $[\alpha / \mathrm{Fe}]$ (each of the ASPCAP $\alpha$-elements) due to uncertainties in $T_{\text {eff }}$. From their fig. 3 , we observe that the most sensible ratio to a $T_{\text {eff }}$ variation is $[\mathrm{O} / \mathrm{Fe}]$. A $\Delta T_{\text {eff }} \sim 200 \mathrm{~K}$ would imply a $\Delta \log g \sim 0.6$ dex in the red giant branch $(\mathrm{RGB})$, and an uncertainty in $[\mathrm{O} / \mathrm{Fe}] \sim 0.25 \mathrm{dex}$ (lower for the other abundances of our interest). The peak of the $\log g$ distribution in the $r<10$ and $r>15 \mathrm{kpc}$ bins shifts towards 0.5 dex lower, approximately. Considering the number of the stars in our furthest bin, we derive that the possible systematic error due to uncertainties in the parameters would lead to underestimate $0.08 \mathrm{dex}$ our $[\mathrm{O} / \mathrm{Fe}]$. However, we detect a larger variation at the most metal-rich side.

The APOGEE sample comprises stars in the RGB and possibly in the asymptotic giant branch (AGB) stages of evolution. These stages are reached by LIMS, producing, at the same time, heavy elements with diverse efficiencies, depending on the initial mass and metallicity of the stars. The photospheres of these stars are enriched mainly by carbon, nitrogen, fluorine, and heavier elements synthesized by the slow neutron capture process (the $s$-process) and by proton-capture nucleosynthesis (the $p$-process). The mixing from the interior (core and shells surrounding the core) to the stellar envelope results in self-pollution of the stellar photosphere.

The abundances of all the $\alpha$-elements analysed in this paper (O, $\mathrm{Mg}, \mathrm{Si}, \mathrm{S}, \mathrm{Ca}, \mathrm{Ti}$ ) are representative of those abundances in the ISM from which the stars formed, because these chemical elements are not synthesized and are not carried to the photosphere of LIMS (see review by Karakas \& Lattanzio 2014). Moreover, half of the other elements studied in this work $(\mathrm{K}, \mathrm{V}, \mathrm{Mn}, \mathrm{Ni})$ are also not generated by LIMS. Therefore, our results for $\mathrm{O}, \mathrm{Mg}, \mathrm{Si}, \mathrm{S}, \mathrm{Ca}, \mathrm{Ti}, \mathrm{K}, \mathrm{V}, \mathrm{Mn}$, $\mathrm{Ni}$ are independent of the evolutionary status of the APOGEE stars.

Nevertheless, C, N, Na, and $\mathrm{Al}$ are produced by LIMS, but in very different proportions. Among these four elements:

(i) $\mathrm{C}$ is the most produced, mainly by low-mass stars $(M<$ $3.5 \mathrm{M}_{\odot}$ ) during the thermal pulses and the third dredge-up (during the AGB);

(ii) $\mathrm{N}$ is the second most produced, mainly by intermediate-mass stars $\left(M>3.5 \mathrm{M}_{\odot}\right)$ during the first and second dredge-ups (during the ascent of the RGB and AGB, respectively) and hot bottom burning;

(iii) $\mathrm{Na}$ and $\mathrm{Al}$ are synthesized mainly by stars with $M>3.5 \mathrm{M}_{\odot}$ during the second and third dredge-ups, with Na more abundantly produced than $\mathrm{Al}$.

Since $\mathrm{C}$ and $\mathrm{N}$ are mainly produced by LIMS, we caution that $\mathrm{C}$ and $\mathrm{N}$ do not reliably represent the original stellar abundances. The $\mathrm{Na}$ and $\mathrm{Al}$ abundances might be enhanced at high Galactocentric radii, because

(i) these elements are produced by intermediate-mass stars in evolved stages (AGB) and consequently by more luminous stars, and

(ii) the APOGEE sample at large $r$ may be more weighted towards these more luminous objects.
However, the $r$-trends of $\mathrm{Na}$ and $\mathrm{Al}$ (Figs 2 and 3) appear to show no enhancement at large radii.

Stars at different distances could also have different age distributions: at the bottom of the giant branch stars may be biased towards a different age distribution than at the upper side, which might affect the overall chemical trends. Nearer stars would be biased towards slightly younger and more metal-rich stars and further stars towards older and more metal-poor ones; however, if it were the case, further stars would show higher $[\alpha / \mathrm{Fe}]$ ratios, because older stars would have formed from an ISM mainly enriched by SNeII. Our analysis reveals the opposite trend with galactocentric distance.

Finally, we estimate the impact of the distance errors in our sample. We assume a normal distribution with uncertainties of 20 percent, and add this noise to the BPG distance values. The resulting fraction of stars with $\left|z_{n}\right|>5$ (after adding the noise) but $|z|<5$ (without noise), with respect to the stars with $|z|>$ 5 , is 40 percent for $r<10 \mathrm{kpc}, 13$ percent for $10<r<15 \mathrm{kpc}$, and 3 percent for $r>15 \mathrm{kpc}$. However, these fractions reduce to 1 percent and lower if we consider stars at $\left|z_{n}\right|<4$. This means that there could be a contamination of $\sim 40$ percent of stars at $4<|z|<5 \mathrm{kpc}$ in our $r<10$ bin. At this $|z|$ range, the density of thin disc stars is negligible, of thick disc stars is $\sim 2$ per cent the density of stars in the plane, and a little less for the density of halo stars. Thus, 50-70 per cent of the contaminant stars, i.e. a $\sim 20$ 30 per cent of the stars in the $r<10 \mathrm{kpc}$ bin, are likely to belong to the thick disc. The resultant median abundances would be dominated by halo stars. Besides, previous works have not found differences in chemistry between thick disc and halo stars. Therefore, we assert that the chemical trends would not be greatly distorted by this contamination.

\section{DISCUSSION}

Differences for a number of the chemical trends with $[M / \mathrm{H}]$ are clear between stars at $r<10$ and $r>15 \mathrm{kpc}$. The lower $\alpha$-elementto-iron ratios found at larger distances are consistent with the low- $\alpha$ population reported during the past decade by a number of workers (e.g. Fulbright 2002; Gratton et al. 2003; Nissen \& Schuster 2010, 2011; Ishigaki, Chiba \& Aoki 2012; Ishigaki, Aoki \& Chiba 2013; Hawkins et al. 2015). From the kinematical properties of their samples, they estimated that the orbits of these stars would place them farther away than stars exhibiting higher $[\alpha / \mathrm{Fe}]$ ratios. This is consistent with our finding (and that of FA15).

Our present study finds a decrease with $r$ consistent with that obtained by FA15 at $r<20 \mathrm{kpc}$. They also observed a considerably larger drop occurring at a Galactocentric radius between $20<r<$ $40 \mathrm{kpc}$, which we cannot confirm in this work, as the APOGEE observations do not extend to cover this distance range.

The trend observed with $[M / \mathrm{H}]$ for the low- $[\alpha / \mathrm{Fe}]$ population has been interpreted in terms of SNeIa, which contribute iron but little $\alpha$-elements. The differences in $[X / F e]$ observed in the present work between stars at $r<10 \mathrm{kpc}$ and $r>15 \mathrm{kpc}$ for the $\alpha$ elements $(\sim 0.1$ dex for $\mathrm{Si}, \mathrm{Ca}$, and $\mathrm{Ti}$, and higher for $\mathrm{O}, \mathrm{Mg}$, and $\mathrm{S})$ are consistent with their expected relative contributions in SNeIa explosions (Tsujimoto et al. 1995) - higher for Si (17 per cent) and $\mathrm{Ca}$ (25 per cent) than for $\mathrm{Mg}, \mathrm{O}$, and $\mathrm{S}$ (negligible).

It is envisioned that this population would form later, during a long period of a relatively slow star formation, from an ISM polluted by both SNeII and SNeIa. The high- $[\alpha / \mathrm{Fe}]$ population would form much earlier, during a short period of rapid star formation, as these stars originated from an ISM enriched mainly by SNeII. If the star formation history presents several bursts, the 
high- $\alpha$ stars would form during the early bursts, while the low- $\alpha$ stars would form at the beginning of later bursts from an ISM contaminated by recent SNeIa, as appears to have occurred in dwarf spheroidal galaxies (see, e.g., Carigi, Hernandez \& Gilmore 2002). Nissen \& Schuster (2010) also proposed that the low- $\alpha$ population could be born in systems which were later accreted into the Milky Way's halo, and which had experienced a long star formation history.

However, the decrease in $[\alpha / \mathrm{Fe}]$ ratios due to the contribution of SNeIa encounters difficulty in explaining the pattern of some abundance ratios, in particular, the decrease observed for $[\mathrm{Ni} / \mathrm{Fe}]$ with metallicity for the low- $\alpha$ population, and the absence of different $[\mathrm{Mn} / \mathrm{Fe}]$ ratios between both populations. These two elements are expected to be released by SNeIa; thus, different patterns should be detected in $\mathrm{Ni}$ and $\mathrm{Mn}$ with respect to iron between stars formed in an ISM enriched by SNeIa and stars which formed from gas without their contribution. In fact, it would be expected that low- $\alpha$ stars would have higher $[\mathrm{Ni} / \mathrm{Fe}]$ and $[\mathrm{Mn} / \mathrm{Fe}]$ than the high- $\alpha$ stars. However, this is not seen either in our work or in previous studies.

A possible explanation was suggested by Kobayashi et al. (2006), who claimed that, for an IMF biased towards stars which explode as low-mass SNIe-II, this would lead to lower $[\alpha / \mathrm{Fe}]$. Kobayashi et al. (2014) also proposed that the nucleosynthesis of 10-20 $\mathrm{M}_{\odot}$ stars could explain the difference in the $[\alpha / \mathrm{Fe}]$ ratios detected in halo stars. Interestingly, McWilliam, Wallerstein \& Mottini (2013) also claimed that a 'top-light' IMF might provide an explanation for the $[\alpha / \mathrm{Fe}]$ and $[\mathrm{Eu} / \mathrm{Fe}]$ deficiencies found in the analysis of the M54 cluster belonging to the Sagittarius dwarf spheroidal galaxy. This possibility would also explain the $[\mathrm{Mn} / \mathrm{Fe}]$ and $[\mathrm{Ni} / \mathrm{Fe}]$ patterns, but it would imply a complex IMF behaviour to explain the different trends - a metallicity-dependent IMF for the low- $\alpha$ stars and a metallicity-independent IMF for the high- $\alpha$ population, as Nissen \& Schuster (2011) indicated.

Another remaining issue is the fact that the nucleosynthetic contribution from AGB stars (the immediate progenitors of the white dwarfs involved in SNeIa explosions) should be detected in the low- $\alpha$ population, if SNeIa have contributed their iron. However, these stars exhibit lower $[\mathrm{Na} / \mathrm{Fe}]$ ratios than the stars presumed to be formed prior to the contributions from SNeIa. As Nissen et al. (2014) speculated, this could be the result if the progenitors of the low- $\alpha$ population were stars of intermediate mass (4-8 $\mathrm{M}_{\odot}$ ), which contribute little $\mathrm{C}$ to the ISM, and even less $\mathrm{Na}$ and $\mathrm{Al}$. The lower median $[\mathrm{Na} / \mathrm{Fe}]$ and $[\mathrm{Al} / \mathrm{Fe}]$ ratios which we found for stars at $r$ $>15 \mathrm{kpc}$ are consistent with a slow chemical-enrichment history. No signature of significant enrichment from AGBs (neither Na nor $\mathrm{Al}$ ) is detected in the distance bin where the low- $\alpha$ population dominates.

The observed increase in $[\mathrm{Al} / \mathrm{Fe}]$ observed for stars in the inner regions can be explained with the assumption of metallicitydependent yields for massive stars (Nomoto, Kobayashi \& Tominaga 2013), and the flat trend with $[M / \mathrm{H}]$ for the farthest stars by the cancellation of the increase in $\mathrm{Al}$ by the even higher contribution of Fe from SNeIa. However, we do not detect an increasing trend of $[\mathrm{Na} / \mathrm{Fe}]$ with $[M / \mathrm{H}]$ in the nearest stars, as observed by Nissen \& Schuster (2010) for the high- $\alpha$ population, which is well explained by the same metallicity-dependent yields also invoked for $\mathrm{Na}$ (Nomoto et al. 2013). Observationally, $\mathrm{Na}$ is determined by APOGEE from relatively weak lines in the $H$ band, yielding less accurate measurements, as suggested by the large scatter detected in our sample as well as in Holtzman et al. (2015), when inspecting $[\mathrm{Na} / \mathrm{Fe}]$ versus $[\mathrm{Fe} / \mathrm{H}]$. For these reasons, our Na results should be considered with caution.
The higher $[X / \mathrm{Fe}]$ ratios at metallicities lower than -1.8 but lower ratios at higher metallicities may be explained by the combination of a top-heavy IMF and a slower SFR in the subsystems accreted by the Galaxy. Recent cosmological simulations predict that massive satellites merging with the host galaxy contribute at smaller radii than low-mass systems (Amorisco 2015). Low-mass systems are expected to experience outflows which release their gas. This would prevent, or at least greatly suppress, subsequent star formation. The expected IMF for low-mass stellar systems at low metallicities is characterized by discontinuities, i.e. a lack of some massive stars (Cerviño 2013). The signature of the few highmass stars in such low-metallicity environments produces stochastic effects on the abundance ratios (Carigi \& Hernández 2008), which could explain the higher dispersion observed in $[X / F e]$ for metalpoor stars.

The stars which we observe today at metallicities lower than -1.8 , born in these low-mass subsystems, would be formed from the nucleosynthetic contribution from a few very massive stars, leading to high ratios with respect to iron at very low metallicities, followed by less massive stars slowly contributing to the ISM where the current stars were born. In contrast, the inner regions would be formed in an ISM which would have reached the same metallicity faster, with the contribution of a larger number of massive stars, although the upper mass limit of the IMF would be lower (Yamada et al. 2013).

\section{CONCLUSIONS}

We have analysed a sample of $\sim 400$ halo stars targeted by the APOGEE survey, located at $|z|>5 \mathrm{kpc}$ from the Galactic plane, and evaluated the chemical trends for the 15 individual abundances determined by ASPCAP. In order to be sure that our trends were not unduly influenced by the estimated distances to our stars, we made use of the available distances estimated by three independent methods for APOGEE stars. Our main conclusions are the following.

(i) An analysis of the elemental abundance $([X / \mathrm{H}])$ variation with distance from the Galactic Centre, $r$, up to the farthest distances observed by APOGEE ( $\sim 20-30 \mathrm{kpc})$, revealed that the chemical trends are almost constant or decrease with $r$. The variation mainly occurred for stars with a global metallicity $[M / \mathrm{H}]>-1.1$.

(ii) We confirmed that the qualitative chemical trends inferred from our data do not depend on the considered distance set.

(iii) The resulting iron abundance trend calculated from the calibrated $[M / \mathrm{H}]$ parameter is constant across the range of $r$ examined, $5 \lesssim r \lesssim 30 \mathrm{kpc}$. The variation for nearer stars measured in our analysis is barely lower than that observed in the previous analysis of in situ halo stars performed by FA15 with SDSS optical spectra at lower resolution. They also reported a larger decrease taking place at $20<r<40 \mathrm{kpc}$. Our evaluation of $[\mathrm{Fe} / \mathrm{H}]$ and $[\mathrm{Ca} / \mathrm{H}]$ is consistent with their results, but we cannot probe the chemical trends at $20<r<40 \mathrm{kpc}$ due to a lack of sample stars in this distance range.

(iv) The median calibrated $[M / \mathrm{H}]$ values, $\sim-1.5$, also agree with previous reports for inner-halo stars (Carollo et al. 2007, 2010; Chen et al. 2014; FA15). The derived MDF from $[M / \mathrm{H}]$ also shows a second peak at $[M / \mathrm{H}] \sim-2.1$, which resembles the median metallicity value associated with the outer-halo population (Carollo et al. 2007, 2010; Beers et al. 2012).

(v) We also evaluated the trends in the abundance ratios relative to iron, $[X / \mathrm{Fe}]$, with distance from the Galactic Centre from in situ stars for 14 chemical elements. This analysis shows that a 
population of stars with different $\alpha$-element enrichment becomes dominant beyond $r \sim 15 \mathrm{kpc}$.

(a) For the $\alpha$-elements, we found significantly lower ratios for more distant stars at metallicities $[M / \mathrm{H}]>-1.1$. We observed a larger separation in these two populations for $[\mathrm{O} / \mathrm{Fe}],[\mathrm{Mg} / \mathrm{Fe}]$, and $[\mathrm{S} / \mathrm{Fe}]$, but all the $\alpha$-elements show a significant decrease of $\gtrsim 0.1$ dex.

Our $[\mathrm{Ca} / \mathrm{Fe}]$ results with $r$ and $[M / \mathrm{H}]$ are consistent with the results reported by FA15 based on SDSS optical spectra. In contrast, we found a decreasing trend of $[\mathrm{Mg} / \mathrm{Fe}]$ with distance, which disagrees with what they observed.

Our results are also consistent with the two different halo populations reported in APOGEE data by Hawkins et al. (2015) at -1.2 $<[M / \mathrm{H}]<-0.55$. The $[\mathrm{O} / \mathrm{Fe}]$ and $[\mathrm{Mg} / \mathrm{Fe}]$ trends we observed are in agreement with their work and other previous reports. Conversely, we found that the two populations also exhibit different $[\mathrm{S} / \mathrm{Fe}]$ ratios.

(b) We find hints of low- $\alpha$ stars having lower $[\mathrm{Ni} / \mathrm{Fe}]$. We detect different $[\mathrm{Mn} / \mathrm{Fe}]$ ratios between the inner and outer regions for the most metal-poor stars $([M / \mathrm{H}]<-1.8)$, although a larger sample of stars at distances farther than $15 \mathrm{kpc}$ and more accurate measurements are necessary to confirm this result.

(c) The $[\mathrm{K} / \mathrm{Fe}]$ trends with $r$ and $[M / \mathrm{H}]$ also provide evidence for different chemical abundance patterns in stars at $r<10 \mathrm{kpc}$ and $r>15 \mathrm{kpc}$.

(d) Both $[\mathrm{Na} / \mathrm{Fe}]$ and $[\mathrm{Al} / \mathrm{Fe}]$ reveal different chemical patterns for the nearer stars compared with the more distant stars. The $[\mathrm{Na} / \mathrm{Fe}]$ ratio exhibits different trends with $r$ and $[M / \mathrm{H}]$ than found for $[\mathrm{Al} / \mathrm{Fe}]$, but the $\mathrm{Na}$ measurements are less reliable. The $[\mathrm{Al} / \mathrm{Fe}]$ ratio increases with metallicity for inner-halo stars, while the more distant stars exhibit a flat trend.

This work corroborates the suggestion that stars with low $[\alpha / \mathrm{Fe}]$ ratios are predominant at larger distances than stars with higher $[\alpha / \mathrm{Fe}]$ ratios, in agreement with previous work which inferred the distances for the low- and high- $\alpha$ populations based on their kinematical properties (Ishigaki et al. 2010; Nissen \& Schuster 2010,2011$)$. The lower $[\alpha / \mathrm{Fe}]$ ratios are consistent with iron enrichment due to $\mathrm{SNeIa}$; the $[\mathrm{Al} / \mathrm{Fe}]$ chemical patterns are also consistent with this hypothesis. The $[\mathrm{Ni} / \mathrm{Fe}]$ and $[\mathrm{Mn} / \mathrm{Fe}]$ exhibit trends with metallicity in both populations which are also consistent with these previous studies. However, their chemical patterns, as well as the lack of signatures of AGB enrichment, are not those expected in a scenario where SNeIa had time to explode. The characteristics of the environments where both populations were formed remain unclear.

In conclusion, the chemical trends inferred for stars ranging over distances from the Galactic Centre of $5<r<30 \mathrm{kpc}$ suggest that, at $r>15 \mathrm{kpc}$, a stellar population begins to dominate which formed with a different chemical-enrichment history than stars at $r<10 \mathrm{kpc}$. Characterization of the different stellar populations with a larger sample of stars will better constrain the IMF and SFR associated with these previous stellar populations. High-quality data for stars at $r$ farther than $15 \mathrm{kpc}$ will help to clarify the chemical properties of the more distant stellar populations in the Galactic halo. Alternatively, the identification of nearby halo stars which probe to large distances (on the basis of their extreme kinematics) will also permit an increase in the number of suitable outer-halo stars for further analysis.

\section{ACKNOWLEDGEMENTS}

EFA acknowledges support from Dirección General de Asuntos del Personal Académico (DGAPA)-Universidad Nacional Autónoma de Méxido (UNAM) postdoctoral fellowships. LC thanks for the financial supports provided by Consejo Nacional de Ciencia y Tecnología (CONACyT) of México (grant 241732), by Programa de Apoyo a Proyectos de Investigación e Innovación Tecnológica (PAPIIT) of México (IG100115, IA100815), and by Ministerio de Economía, Industria y Competitividad (MINECO) of Spain (AYA2010-16717). TCB acknowledges partial support for this work from grants PHY 08-22648; Physics Frontier Center/Joint Institute for Nuclear Astrophysics (JINA), and PHY-1430152; Physics Frontier Center/JINA Center for the Evolution of the Elements (JINACEE), awarded by the US National Science Foundation. Funding for SDSS-III has been provided by the Alfred P. Sloan Foundation, the Participating Institutions, the National Science Foundation, and the US Department of Energy Office of Science. The SDSS-III website is http://www.sdss3.org/.

SDSS-III is managed by the Astrophysical Research Consortium for the Participating Institutions of the SDSS-III Collaboration including the University of Arizona, the Brazilian Participation Group, Brookhaven National Laboratory, University of Cambridge, Carnegie Mellon University, University of Florida, the French Participation Group, the German Participation Group, Harvard University, the Instituto de Astrofisica de Canarias, the Michigan State/Notre Dame/JINA Participation Group, Johns Hopkins University, Lawrence Berkeley National Laboratory, Max Planck Institute for Astrophysics, Max Planck Institute for Extraterrestrial Physics, New Mexico State University, New York University, Ohio State University, Pennsylvania State University, University of Portsmouth, Princeton University, the Spanish Participation Group, University of Tokyo, University of Utah, Vanderbilt University, University of Virginia, University of Washington, and Yale University.

\section{REFERENCES}

Alam S. et al., 2015, ApJS, 219, 12

Allende Prieto C., Beers T. C., Wilhelm R., Newberg H. J., Rockosi C. M., Yanny B., Lee Y. S., 2006, ApJ, 636, 804

Allende Prieto C. et al., 2014, A\&A, 568, A7

Amorisco N. C., 2015, MNRAS, 450, 575

An D. et al., 2013, ApJ, 763, 65

An D., Beers T. C., Santucci R. M., Carollo D., Placco V. M., Lee Y. S., Rossi S., 2015, ApJ, 813, L28

Beers T. C. et al., 2012, ApJ, 746, 34

Belokurov V. et al., 2009, MNRAS, 397, 1748

Binney J. et al., 2014, MNRAS, 437, 351

Bovy J. et al., 2014, ApJ, 790, 127

Bressan A., Marigo P., Girardi L., Salasnich B., Dal Cero C., Rubele S., Nanni A., 2012, MNRAS, 427, 127

Burnett B., Binney J., 2010, MNRAS, 407, 339

Burnett B. et al., 2011, A\&A, 532, A113

Carigi L., Hernández X., 2008, MNRAS, 390, 582

Carigi L., Hernandez X., Gilmore G., 2002, MNRAS, 334, 117

Carollo D. et al., 2007, Nature, 450, 1020

Carollo D. et al., 2010, ApJ, 712, 692

Cerviño M., 2013, New Astron. Rev., 57, 123

Chen Y. Q., Zhao G., Carrell K., Zhao J. K., Tan K. F., Nissen P. E., Wei P., 2014, ApJ, 795, 52

Côté B., Ritter C., O’Shea B. W., Herwig F., Pignatari M., Jones S., Fryer C. L., 2016, ApJ, 824, 82

Dawson K. S. et al., 2013, AJ, 145, 10 
Eisenstein D. J. et al., 2011, AJ, 142, 72

Fernández-Alvar E. et al., 2015, A\&A, 577, A81 (FA15)

Fernández-Trincado J. G. et al., 2016, ApJ, preprint (arXiv:1604.01279)

Fulbright J. P., 2002, AJ, 123, 404

García Pérez A. E. et al., 2016, AJ, 151, 144

Ghez A. M. et al., 2008, ApJ, 689, 1044

Gratton R. G., Carretta E., Desidera S., Lucatello S., Mazzei P., Barbieri M., 2003, A\&A, 406, 131

Gunn J. E. et al., 2006, AJ, 131, 2332

Hawkins K., Jofré P., Masseron T., Gilmore G., 2015, MNRAS, 453, 758

Hayden M. R. et al., 2015, ApJ, 808, 132 (H15)

Holtzman J. A. et al., 2015, AJ, 150, 148

Ishigaki M., Chiba M., Aoki W., 2010, PASJ, 62, 143

Ishigaki M. N., Chiba M., Aoki W., 2012, ApJ, 753, 64

Ishigaki M. N., Aoki W., Chiba M., 2013, ApJ, 771, 67

Janesh W. et al., 2016, ApJ, 816, 80

Karakas A. I., Lattanzio J. C., 2014, PASA, 31, e030

Kobayashi C., Umeda H., Nomoto K., Tominaga N., Ohkubo T., 2006, ApJ, 653,1145

Kobayashi C., Ishigaki M. N., Tominaga N., Nomoto K., 2014, ApJ, 785, L5

Lind K. et al., 2015, A\&A, 575, L12

McWilliam A., Wallerstein G., Mottini M., 2013, ApJ, 778, 149

Majewski S. R. et al., 2015, AJ, preprint (arXiv:1509.05420)

Marigo P., Girardi L., Bressan A., Groenewegen M. A. T., Silva L., Granato G. L., 2008, A\&A, 482, 883
Mészáros S. et al., 2015, AJ, 149, 153

Nidever D. L. et al., 2015, AJ, 150, 173

Nissen P. E., Schuster W. J., 2010, A\&A, 511, L10

Nissen P. E., Schuster W. J., 2011, A\&A, 530, A15

Nissen P. E., Chen Y. Q., Carigi L., Schuster W. J., Zhao G., 2014, A\&A, 568, A25

Nomoto K., Kobayashi C., Tominaga N., 2013, ARA\&A, 51, 457

Pinsonneault M. H. et al., 2014, ApJS, 215, 19

Santiago B. X. et al., 2016, A\&A, 585, A42

Schultheis M. et al., 2014, AJ, 148, 24 (S14)

Tissera P. B., Beers T. C., Carollo D., Scannapieco C., 2014, MNRAS, 439, 3128

Tsujimoto T., Nomoto K., Yoshii Y., Hashimoto M., Yanagida S., Thielemann F.-K., 1995, MNRAS, 277, 945

Yamada S., Suda T., Komiya Y., Aoki W., Fujimoto M. Y., 2013, MNRAS, 436,1362

Yanny B. et al., 2009, AJ, 137, 4377

York D. G. et al., 2000, AJ, 120, 1579

Zacharias N., Finch C. T., Girard T. M., Henden A., Bartlett J. L., Monet D. G., Zacharias M. I., 2013, AJ, 145, 44

Zasowski G. et al., 2013, AJ, 146, 81

This paper has been typeset from a $\mathrm{T}_{\mathrm{E}} \mathrm{X} / \mathrm{L} \mathrm{T}_{\mathrm{E}} \mathrm{X}$ file prepared by the author. 